\title{
FREE BOUNDARY PROBLEMS INVOLVING SINGULAR WEIGHTS
}

\author{
JIMMY LAMBOLEY, YANNICK SIRE, AND EDUARDO V. TEIXEIRA
}

\begin{abstract}
In this paper we initiate the investigation of free boundary minimization problems ruled by general singular operators with $A_{2}$ weights. We show existence, boundedness and continuity of minimizers. The key novelty is a sharp $C^{1+\gamma}$ regularity result for solutions at their singular free boundary points. We also show a corresponding non-degeneracy estimate.
\end{abstract}

\section{Contents}

1. Introduction

2. Mathematical set-up

3. Existence and local boundedness 6

4. Universal Continuity 7

5. Homogenization 11

6. $C^{1+\gamma}$ regularity at the free boundary 14

7. Nondegeneracy and weak geometry 16

\begin{tabular}{ll} 
References & 19 \\
\hline
\end{tabular}

\begin{abstract}
Aknowledgement: The authors would like to thank the hospitality of the Univ. Federal do Ceara in Fortaleza, where this work was initiated, and the Brazilian-French Network in Mathematics as well. This work was also supported by the projects ANR-18-CE40-0013 SHAPO financed by the French Agence Nationale de la Recherche (ANR) and the Brazilian National Council for Scientific and Technological Development,CNPq, under MCTI/CNPQ/Universal 14/2014 - Faixa C project No 441632/2014-9. Y.S. would like to thank the Simons foundation.
\end{abstract}

2010 Mathematics Subject Classification. Primary 35B65. Secondary 35J60, 35J70.

Key words and phrases. Free boundary problems, Muckenhoupt weights, geometric regularity. 


\section{INTRODUCTION}

We study local minimizers of singular, discontinuous functionals of the form

$$
\mathcal{J}(u, \Omega)=\int_{\Omega}\left(\omega(x)|\nabla u|^{2}+\chi_{\{u>0\}}\right) d x \longrightarrow \min ,
$$

where $\Omega$ is a bounded domain of $\mathbb{R}^{d}, d \geq 2$, and $\omega(x)$ is a measurable, singular $A_{2}$ weight in the sense that $0 \leq \omega \leq+\infty$, both $\omega$ and $\omega^{-1}$ are locally integrable, and

$$
\left(\int_{B_{r}\left(z_{0}\right)} \omega\right)\left(\int_{B_{r}\left(z_{0}\right)} \omega^{-1}\right) \leq C r^{2 d},
$$

for all balls $B_{r}\left(z_{0}\right) \subset \Omega$. Thus, $\omega$ may become zero or infinity along a lower-dimensional subset of $\Omega$, hereafter denoted by:

$$
\Lambda_{0}(\omega):=\omega^{-1}(0), \quad \Lambda_{\infty}(\omega):=\omega^{-1}(+\infty) .
$$

It will also be convenient to denote $\Lambda(\omega):=\Lambda_{0}(\omega) \cup \Lambda_{\infty}(\omega)$. The class of $A_{2}$ weight functions was introduced by Muckenhoupt [13] and is of central importance in modern harmonic analysis and its applications. A canonical example of an $A_{2}$ function is $|x|^{\alpha}$ with $-d<\alpha<d-$ having an isolated singularity at the origin. Recent results, see for instance [3], show that $A_{2}$ weights play an important role in the theory of non-local diffusive problems. In particular, when $\omega(x)=\left|x_{1}\right|^{\beta},-1<\beta<1$, and the axis $\left\{x_{1}=0\right\}$ is a subset of $\partial \Omega$, then (1.1) falls into the case studied in [2], where fractional cavitation problems are considered.

The mathematical analysis of free boundary cavitation problems goes back to the pioneering work of Alt and Caffarelli [1], corresponding to the case $\omega \equiv 1$ in (1.1). In the present work, we start the investigation of free boundary problems of the type (1.1), for possibly singular weights, that is when $\Lambda_{\infty}(\omega) \neq \emptyset$. Similarly, we can treat the degenerate case, i.e. when $\Lambda_{0}(\omega) \neq \emptyset$. However, for didactical purposes, in this paper we shall restrict the analysis to singular weights.

We show existence of a local minimizer $u$, and analyze analytic and weak geometric properties of the free boundary $\partial\{u>0\} \cap \Omega$. The latter task is, in principle, a delicate issue. For instance, one notices, for singular weights, the existence of two distinct types of free boundary points:

(1) $\partial\{u>0\} \cap \Lambda_{\infty}=\emptyset$;

(2) $\partial\{u>0\} \cap \Lambda_{\infty} \neq \emptyset$.

Case (1) refers to a non-homogeneous version of the Alt-Caffarelli problem, 1], as, away from the singular set, the weight is uniformly elliptic, see for instance 4, 5, 9, 14. Case (2) is rather more delicate, and in particular, a central result we prove in this current work classifies the geometric behavior of a local minimizer near a free boundary point $z_{0} \in \partial\{u>0\} \cap \Lambda_{\infty}$, 
in terms of its singularity rate near $z_{0}$ - a purely analytic information of the problem. Indeed, we show local minimizers are precisely $C^{1+\gamma}$ smooth along their corresponding free boundaries, where $\gamma$ is half of the geometric blow-up rate of $\omega$ as it approaches the singular set $\Lambda_{\infty}$; see condition (H2) for precise definitions.

The paper is organized as follows. In Section 2 we formally present the minimization problem we shall study. A brief description of the initial mathematical tools required in the investigation of $A_{2}$-singular free boundary problems is also delivered in that section. In Section 3 we discuss existence and $L^{\infty}$ bounds for minimizers, whereas in Section 4 we establish various compactness properties for family of minimizers. Section 5 is devoted to some considerations related to homogenization. In Section 6 we prove the key novel result of the paper, namely that solutions to $A_{2}$ cavitation problems are $C^{1+\gamma}$ regular at their singular free boundary points, where $\gamma$ is a sharp prescribed value. In particular, if $z_{0}$ is a free boundary point and $\omega\left(z_{0}\right)<+\infty$, that is it is non-singular, then $\gamma\left(z_{0}\right)=0$ and we recover the classical Alt-Caffarelli Lipschitz regularity estimate for cavitation problems. Finally in Section 7 we obtain a quantitative non-degeneracy estimate for solutions near their singular free boundary points.

\section{Mathematical Set-UP}

In this section we give a precise description of the minimization problem considered in this article and gather some of the main known results about elliptic equations involving $A_{2}$ weights, required in our study.

Given an open set $\Omega \subset \mathbb{R}^{d}$, we denote by $\mathcal{M}(\Omega)$ the set of all real-valued measurable functions defined on $\Omega$. A nonnegative locally integrable function $\omega: \Omega \rightarrow \mathbb{R}$ is said to be an $A_{2}$ weight if $\omega^{-1}$ is also locally integrable and

$$
\sup _{B \subset \Omega}\left(\frac{1}{|B|} \int_{B} \omega\right)\left(\frac{1}{|B|} \int_{B} \omega^{-1}\right) \leq C_{1},
$$

holds for a constant $C_{1}>0$ and any ball $B \subset \Omega$. Two weights are said to belong to the same $A_{2}$ class if condition (2.1) is verified for both functions with the same constant $C_{1}$.

For an $A_{2}$ weight $\omega$ and $1 \leq p<\infty$, we define

$$
L^{p}(\Omega, \omega)=\left\{f \in \mathcal{M}(\Omega) \mid\|f\|_{L^{p}(\Omega, \omega)}:=\left(\int_{\Omega}|f(x)|^{p} \omega(x) d x\right)^{1 / p}<\infty\right\} .
$$

Accordingly, we define the weighted Sobolev space as

$$
W^{1, p}(\Omega, \omega):=\left\{u \in L^{p}(\Omega, \omega) \mid D_{i} u \in L^{p}(\Omega, \omega), \text { for } i=1,2, \cdots d\right\},
$$

and, by convention, we write $W^{1,2}(\Omega, \omega)$ as $H^{1}(\Omega, \omega)$.

An $A_{2}$ weight $\omega$ gives raise to the degenerate/singular elliptic operator

$$
L_{\omega}(\cdot)=\operatorname{div}(\omega \nabla \cdot),
$$


which acts on $H^{1}(\Omega, \omega)$. In a series of three papers, 6, 7, 8], Fabes, Jerison, Kenig, and Serapioni developed a systematic theory for this class of operators: existence of weak solutions, Sobolev embeddings, Poincaré inequality, Harnack inequality, local solvability in Hölder spaces, and estimates on the Green's function. Below we state four results from [6], see also [11] supporting, directly or indirectly, the framework developed in this present article.

Theorem 1 (Solvability in Sobolev spaces). Let $\Omega \subset \mathbb{R}^{d}$ be a smooth bounded domain, $h=\left(h_{1}, \ldots, h_{n}\right)$ satisfying $|h| / \omega \in L^{2}(\Omega, \omega)$, and $g \in$ $H^{1}(\Omega, \omega)$. Then, there exists a unique solution $u \in H^{1}(\Omega, \omega)$ of $L_{\omega} u=$ $-\operatorname{div} h$ in $\Omega$ with $u-g \in H_{0}^{1}(\Omega, \omega)$.

Theorem 2 (Local Hölder regularity). Let $h$ be a weak solution of $L_{\omega} h=0$ in $B_{1} \subset \mathbb{R}^{d}$. Then, $u$ is Hölder continuous in $B_{1 / 2}$ with a Hölder exponent $\mu$ depending only on $d$ and the $A_{2}$ class of $\omega$. Furthermore

$$
\|h\|_{C^{\mu}\left(B_{1 / 2}\right)} \leq M\left(\frac{1}{\omega\left(B_{1}\right)} \int h^{2}(x) \omega(x) d x\right)^{1 / 2},
$$

for a constant $M$ also depending only on $d$ and the $A_{2}$ class of $\omega$.

Theorem 3 (Harnack inequality). Let $u$ be a positive solution of $L_{\omega} u=0$ in $B_{4 R}\left(x_{0}\right) \subset \mathbb{R}^{d}$. Then, $\sup _{B_{R}\left(x_{0}\right)} u \leq C \inf _{B_{R}\left(x_{0}\right)} u$ for some constant $C$ depending only on $d$ and the $A_{2}$ class of $\omega$ - and in particular, independent of $R$.

Theorem 4 (Poincaré inequality). There is as positive constant $C>1$ such that for all function $f$ in $H^{1}\left(B_{R}, \omega\right)$ satisfying $f=0$ on $\partial B_{R}$, the following holds

$$
\int_{B_{R}}|f|^{2} \omega d x \leq C R^{2} \int_{B_{R}}|\nabla f|^{2} \omega d x .
$$

Let us now turn to the mathematical description of the problem to be studied in the present article. Given a nonnegative boundary datum $f \in$ $H^{1}(\Omega, \omega) \cap L^{\infty}(\Omega)$, we consider the minimization problem

$$
\mathcal{J}(\omega, u, \Omega)=\int_{\Omega}\left(\omega(x)|\nabla u|^{2}+\chi_{\{u>0\}}\right) d x \longrightarrow \min ,
$$

among functions $u \in H_{f}^{1}(\Omega, \omega):=f+H_{0}^{1}(\Omega, \omega)$, where $\chi_{O}$ stands for the characteristic function of the set $O$ and $\omega$ is an $A_{2}$ weight.

We are mostly interested in local geometric properties of local minima near a singular free boundary point. Henceforth, as to properly carry out the analysis, it is convenient to localize the problem into the unit ball $B_{1}$ and assume the origin is a free boundary point, i.e., $0 \in \partial\{u>0\}$. In addition we shall assume throughout the paper the following structural condition on the weight $\omega$ :

(H1) The weight $\omega$ belongs to $A_{2}$-class and $0<\tau_{0} \leq \omega \leq+\infty$ a.e. in $\Omega$. 
It turns out that $(\mathrm{H} 1)$ prescribes minimal condition under which one can develop an existence and regularity theory for corresponding singular cavitation problem.

We comment that we have chosen to develop the analysis of problems involving singular weights, rather than degenerate ones. In turn, we consider strictly positive weights that may blow-up along its singular set $\Lambda_{\infty}(\omega)$. We could similarly treat bounded, degenerate weights of order $\gtrsim r^{\sigma}$, for some $\sigma<2$.

A number of physical free boundary problems fall into the above mathematical set-up. Typical examples of weight functions we have in mind are

$$
\omega(x)=\left|x^{\prime}\right|^{\alpha},
$$

where $x=\left(x^{\prime}, x_{m+1}, \cdots, x_{d}\right)$, for $0 \leq m<d$ and $-m<\alpha \leq 0$. More generally, if $\mathcal{N}$ is an $m$-dimensional manifold properly embedded in $\mathbb{R}^{d}$, $0 \leq m<d$, we are interested in weights of the form

$$
\omega(x)=\operatorname{dist}(x, \mathcal{N})^{\alpha},
$$

for some $-m<\alpha \leq 0$. This class of weight functions gives raise to the analysis of free boundary problems ruled by diffusion operators with an $m$-dimensional singular set. Anisotropic weights of the form

$$
\omega(x):=\prod_{i=1}^{d}\left|x_{i}\right|^{\alpha_{i}},
$$

also fall under the hypothesis considered in this work. In this case, condition (H1) is verified as long as $-1<\alpha_{i} \leq 0$.

We are further interested in weights with possible distinct behaviors along sets of different dimensions, say

$$
\omega(x)=\left|\left(x_{1}, x_{2}, \cdots, x_{m}\right)\right|^{\alpha_{1}} \cdot\left|\left(x_{m+1}, \cdots, x_{d}\right)\right|^{\alpha_{2}},
$$

where $-m<\alpha_{1} \leq 0,-d+m<\alpha_{2} \leq 0$. In this model, 0 is a singular free boundary point of degree $\left|\alpha_{1}+\alpha_{2}\right|$, as we shall term later. If we label the cones $\mathcal{C}_{1}:=\left\{\Pi_{i=1}^{m} x_{i}=0\right\}$ and $\mathcal{C}_{2}:=\left\{\Pi_{i=m+1}^{d} x_{i}=0\right\}$, then any free boundary point in $\mathcal{C}_{1} \backslash \mathcal{C}_{2}$ is singular of degree $\left|\alpha_{1}\right|$ and similarly, a point in $\partial\{u>0\} \cap\left(\mathcal{C}_{2} \backslash \mathcal{C}_{1}\right)$ is singular of degree $\left|\alpha_{2}\right|$. Any other free boundary point $z \in \partial\{u>0\} \backslash\left(\mathcal{C}_{1} \cup \mathcal{C}_{2}\right)$ is a free boundary point of degree 0 .

Notice that no continuity assumption has been required on $\omega$. In particular, we are interested in the analysis of free boundary problems in possibly random media. Given a measurable function $0<c_{0} \leq \theta<c_{0}^{-1}$ defined on the unit sphere $\mathbb{S}^{d-1}, 0 \leq m<d-2$ and $-m<\alpha \leq 0$, we can always define an $\alpha$-homogeneous, $A_{2}$ weight function $\omega$ in $B_{1} \backslash\{0\}$ as

$$
\omega(x):=\left|x^{\prime}\right|^{\alpha} \cdot \theta\left(\frac{x}{|x|}\right),
$$


where, as above, $x=\left(x^{\prime}, x_{m+1}, \cdots, x_{d}\right)$. This is another important example of weights we have in mind as to motivate this work.

We conclude this section by setting a nomenclature convention: hereafter any constant that depends only upon dimension, $d$, and $\omega$ will be called universal.

\section{EXISTENCE AND LOCAL BOUNDEDNESS}

In this preliminary section, we discuss existence and local boundedness of minimizers. We also comment on the Euler-Lagrange equation satisfied by a local minimum. The proofs follow somewhat classical arguments, thus we simply sketch them here for sake of completeness.

Given a nonnegative boundary datum $f \in H^{1}(\Omega, \omega)$, one can consider a minimizing sequence $v_{j} \in H_{f}^{1}(\Omega, \omega)$. Clearly,

$$
C>\mathcal{J}\left(v_{j}\right) \geq \int_{\Omega} \omega(x)\left|\nabla v_{j}\right|^{2} d x .
$$

Thus, up to a subsequence, $v_{j} \rightarrow u$ weakly in $H^{1}(\Omega, \omega)$, for some function $u \in H_{f}^{1}(\Omega, \omega)$. By compactness embedding, $v_{j} \rightarrow u$ in $L^{2}(\Omega, \omega)$ and $v_{j} \rightarrow u$ a.e. in $\Omega$. Passing to the limit as $j \rightarrow \infty$ (see [1, Section 1.3] to handle the term $\left.\chi_{\left\{v_{j}>0\right\}}\right)$, one concludes

$$
\mathcal{J}(u) \leq \liminf _{j \rightarrow \infty} \mathcal{J}\left(v_{j}\right)=\min \mathcal{\partial} .
$$

This shows the existence of a minimizer. To verify that $u$ is nonnegative, one simply compares $u$ with $u^{+}$in the minimization problem. Also, if the boundary datum $f$ is assumed to be in $H^{1}(\Omega, \omega) \cap L^{\infty}(\Omega)$, then for each $|t| \ll 1$, the function $u+t\left(\|f\|_{\infty}-u\right)^{-}$competes with $u$ in the minimization problem. Standard computations yield $\left\{u>\|f\|_{\infty}\right\}$ has measure zero, that is, $\left\{0 \leq u \leq\|f\|_{\infty}\right\}$ has total measure in $\Omega$.

Now, if $\varphi$ is a nonnegative test function in $C_{0}^{\infty}(\Omega)$, then $u+\varphi$ competes with $u$ in the minimization problem. As $\{u+\varphi>0\} \supset\{u>0\}$, there holds,

$$
-2 \int_{\Omega} \omega(x) \nabla u \cdot \nabla \varphi d x=\mathcal{J}(u+\varphi)-\mathcal{J}(u)-\int_{\Omega}\left(\chi_{\{u+\varphi>0\}}-\chi_{\{u>0\}}\right) d x \geq 0 .
$$

This shows $\operatorname{div}(\omega(x) \nabla u)$ defines a nonnegative measure $\nu$. If $B_{\delta}\left(x_{0}\right) \subset\{u>$ $0\}$, then given a test function $\varphi \in C_{0}^{\infty}\left(B_{\delta}\left(x_{0}\right)\right)$, for $0<|t| \ll 1, u+t \varphi$ is also positive in $B_{\delta}\left(x_{0}\right)$, thus we conclude $\nu \equiv 0$ in the interior of $\{u>0\}$.

Let us gather the information delivered above and state as a theorem for future reference.

Theorem 5. Let $\omega$ be any $A_{2}$ weight and $f \in H^{1}(\Omega, \omega)$ nonnegative. Then there exists a minimizer $u \in H^{1}(\Omega, \omega)$ to problem (2.2) such that $u=f$ on $\partial \Omega$, in the trace sense. Furthermore, $u$ is nonnegative, $\|u\|_{L^{\infty}(\Omega)} \leq$ 
$\|f\|_{L^{\infty}(\Omega)}$, and there exists a non-negative Radon measure $\nu$, supported on its free boundary $\partial\{u>0\}$, such that

$$
\operatorname{div}(\omega(x) \nabla u)=\nu
$$

is verified in the distributional sense.

\section{Universal Continuity}

In this section we establish that local minima of $\mathcal{J}$ are locally Hölder continuous, with universal bounds. In particular, any family of bounded local minima is pre-compact in the uniform convergence topology. Hereafter in this section we shall use the notation

$$
f_{\left.B_{r}(y)\right)} f(x) \omega(x) d x:=\frac{1}{\omega\left(B_{r}(y)\right)} \int_{B_{r}(y)} f(x) \omega(x) d x .
$$

We also write, for a measurable set $A, \omega(A):=\int_{A} \omega(x) d x$. Recall $A_{2^{-}}$ weights are doubling measures, i.e. there exists $D>0$ such that

$$
0<\omega\left(B_{2 r}(y)\right) \leq D \omega\left(B_{r}(y)\right),
$$

for all $y$ and $r>0$, see for instance [11].

We start off by investigating the scaling of the problem. Let $u$ be a local minimizer of

$$
\mathcal{J}\left(\omega, u, B_{1}\right):=\int_{B_{1}} \omega(x)|\nabla u(x)|^{2}+\chi_{\{u>0\}} d x .
$$

For $x_{0} \in B_{1 / 2}, 0<\rho<1 / 2$ and $t>0$, define the re-scaled function $\tilde{u}: B_{1} \rightarrow$ $\mathbb{R}$ by

$$
\tilde{u}(y)=t \cdot u\left(x_{0}+\rho y\right) .
$$

If we define the map $\Phi: B_{1} \rightarrow B_{\rho}\left(x_{0}\right)$ as $z=\Phi(y)=x_{0}+\rho y$, for a subdomain $\Omega \subset \Phi\left(B_{1}\right)$, change of variables yields

$$
\begin{aligned}
\mathcal{J}(\omega, u, \Omega) & =\int_{\Phi^{-1}(\Omega)}\left[\omega\left(x_{0}+\rho y\right)\left|\nabla u\left(x_{0}+\rho y\right)\right|^{2}+\chi_{\left\{u\left(x_{0}+\rho y\right)>0\right\}}\right] \rho^{d} d y \\
& =\int_{\Phi^{-1}(\Omega)}\left[\omega\left(x_{0}+\rho y\right) t^{-2} \rho^{-2}|\nabla \tilde{u}|^{2}+\chi_{\{\tilde{u}>0\}}\right] \rho^{d} d z \\
& =\rho^{d-2} t^{-2} \int_{\Phi^{-1}(\Omega)}\left[\omega\left(x_{0}+\rho y\right)|\nabla \tilde{u}|^{2}+t^{2} \rho^{2} \chi_{\{\tilde{u}>0\}}\right] d z
\end{aligned}
$$

Hence, we conclude that $\tilde{u}$ is a local minimizer of a functional $\tilde{\mathcal{J}}$ ruled by a weighted function $\tilde{\omega}$ in the same $A_{2}$ class of $\omega$, but with jump forcing term $t^{2} \rho^{2} \chi_{\{\tilde{u}>0\}}$.

Given a local bounded minimizer $u$ of $\mathcal{J}$ in $B_{1}$, for a fixed $0<\varepsilon<1$, select

$$
\rho=\sqrt{\varepsilon f_{B_{1}} u^{2} \omega d x} \text { and in the sequel } t=\rho^{-1} \sqrt{\varepsilon} \text {. }
$$


The corresponding scaled function $\tilde{u}$ then verifies $f_{B_{1}} \tilde{u}^{2} \omega d x \leq 1$ and is a local minimizer of

$$
\mathcal{f}^{\varepsilon}(v):=\int \tilde{\omega}(x)|\nabla v(x)|^{2}+\varepsilon \chi_{\{v>0\}} d x,
$$

where $\tilde{\omega}(x)$ lies in the same $A_{2}$ class as $\omega$.

Notice that proving Hölder continuity for $u$ is equivalent to proving $\tilde{u}$ is Hölder continuous; any estimate proven for $\tilde{u}$ yields a corresponding one for $u$, with proper constants adjustments, depending only on the choices for $t$ and $\rho$. In particular, should these choices be universal, the corresponding result for $u$ will be also universal.

The above discussion motivates the following result, which measures how much a local minimizer of a functional with small jumping force deviates from its $\omega$-harmonic replacement. We shall need to work with a slightly more general class of operators.

Proposition 6. Let $\beta: \mathbb{R} \rightarrow[0,1]$ be a measurable function and $u$ be a local minimizer of

$$
\mathcal{g}^{\varepsilon, \beta}(v)=\int \omega(x)|\nabla v(x)|^{2}+\varepsilon \beta(v) d x,
$$

where $\omega$ is in the $A_{2}$-class. Let $h$ be the unique weak solution of

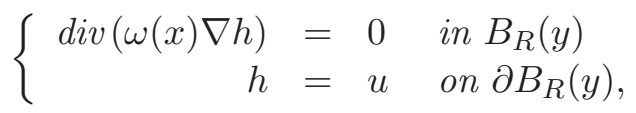

where $y \in B_{1 / 2}$ and $0<R<1 / 2$. Then

$$
\int_{B_{R}(y)} \omega(x)|\nabla u(x)-\nabla h(x)|^{2} d x \leq \varepsilon R^{d} .
$$

Proof. Since $h$ competes with $u$ in the minimization problem,

$$
\mathcal{J}^{\varepsilon, \beta}(u) \leq \mathcal{J}^{\varepsilon, \beta}(h),
$$

which yields

$$
\int_{B_{R}(y)} \omega(x)\left(|\nabla u(x)|^{2}-|\nabla h(x)|^{2}\right) d x \leq \varepsilon \int_{B_{R}(y)}(\beta(h)-\beta(u)) d x \leq \varepsilon R^{d} .
$$

Now, from the PDE satisfied by $h$, we have

$$
\int_{B_{R}(y)} \omega(x) \nabla h \cdot \nabla(h-u) d x=0
$$

hence,

$$
\int_{B_{R}(y)} \omega(x)|\nabla u-\nabla h|^{2} d x=\int_{B_{R}(y)} \omega(x)\left(|\nabla u|^{2}-|\nabla h|^{2}\right) d x,
$$

and the Proposition is proven. 
Finally from Theorem 2, $\omega$-harmonic functions are locally Hölder continuous. More precisely, if $h$ is a weak solution of $\operatorname{div}(\omega(x) \nabla h)=0$ in $B_{1 / 2}$, and say

$$
\left(f_{B_{1 / 2}} h^{2}(x) \omega(x) d x\right)^{1 / 2} \leq 1
$$

then for some $0<\mu<1$ and $\Lambda$, depending only on dimension and $A_{2}$ class of $\omega$ there holds

$$
\|h\|_{C^{0, \mu}\left(B_{1 / 4}\right)} \leq \Lambda \text {. }
$$

In what follows we shall argue along the lines of [16].

Lemma 7. Assume (H1). There exist universal constants $0<\varepsilon<1$, $0<\lambda<1 / 4$ and $\left|a_{0}\right|<\Lambda$ such that if $u$ is a local minimizer of $\mathrm{g}^{\varepsilon, \beta}$, satisfying $f_{B_{1}}|u|^{2} \omega(x) d x \leq 1$, then

$$
f_{B_{\lambda}}\left|u-a_{0}\right|^{2} \omega(x) d x \leq \lambda^{\mu}
$$

Proof. Let $h$ be the $\omega$-harmonic replacement of $u$ in $B_{1 / 2}$. From Proposition 6] and Poincaré inequality, we can estimate

$$
\int_{B_{1 / 2}}|u-h|^{2} \omega(x) d x \leq C \varepsilon
$$

Because $f_{B_{1}}|u|^{2} \omega(x) d x \leq 1$, we can make a first choice on the smallness of $\varepsilon$ to ensure

$$
f_{B_{1 / 2}} h^{2}(x) \omega(x) d x \leq \sqrt{(D+1)}
$$

where $D>0$ is the doubling constant from (4.2). Indeed,

$$
\begin{aligned}
\|h\|_{L^{2}\left(B_{1 / 2}, \omega\right)} & \leq\|h-u\|_{L^{2}\left(B_{1 / 2}, \omega\right)}+\|u\|_{L^{2}\left(B_{1 / 2}, \omega\right)} \\
& \leq \sqrt{C \varepsilon}+\|u\|_{L^{2}\left(B_{1}, \omega\right)} \\
& \leq \sqrt{C \varepsilon}+\sqrt{\omega\left(B_{1}\right)} \\
& \leq \sqrt{C \varepsilon}+\sqrt{D \omega\left(B_{1 / 2}\right)} \\
& \leq \sqrt{(D+1) \omega\left(B_{1 / 2}\right)}
\end{aligned}
$$

provided $\varepsilon>0$ is small enough. We now set $a_{0}=h(y)$ and, for a $0<\lambda<1 / 4$ to be chosen, estimate

$$
\begin{aligned}
f_{B_{\lambda}}\left|u-a_{0}\right|^{2} \omega(x) d x & \leq 2\left(f_{B_{\lambda}}|h-h(y)|^{2} \omega(x) d x+f_{B_{\lambda}}|u-h|^{2} d x\right) \\
& \leq 2\left(\Lambda(D+1) \cdot \lambda^{2 \mu}+\frac{C \varepsilon \omega\left(B_{1}\right)}{\omega\left(B_{\lambda}\right)}\right) .
\end{aligned}
$$

Now we choose $0<\lambda \ll 1$ so small that

$$
\Lambda(D+1) \cdot \lambda^{2 \mu}=\frac{1}{4} \lambda^{\mu}
$$


and in the sequel select $\varepsilon>0$ such that

$$
\frac{C \varepsilon \omega\left(B_{1}\right)}{\omega\left(B_{\lambda}\right)}=\frac{1}{4} \lambda^{\mu}
$$

Note these choices are universal. Lemma 7 is proven.

We are ready to prove universal Hölder continuity for local minimizers.

Theorem 8 (Local $\left.C^{0, \tau}\right)$. Let $u$ be local minimizer of $\mathcal{J}$ in $B_{1}$. There exist universal constants $0<\tau \ll 1$ and $C>1$ such that $u \in C^{0, \tau}\left(B_{1 / 2}\right)$ and

$$
\|u\|_{C^{0, \tau}\left(B_{1 / 2}\right)} \leq C\|u\|_{L^{2}\left(B_{1}\right)} \text {. }
$$

Proof. The idea is to iterate Lemma 7 . Let $u$ be a minimizer of $\mathcal{g}^{\varepsilon}$, where $\varepsilon>0$ is the number from Lemma 7. Note that from the discussion at the beginning of this Section, proving Hölder continuity for a local minimizer of $\mathcal{J}^{\varepsilon}$ yields corresponding estimates for minimizers of the original functional J.

Define $u_{1}: B_{1} \rightarrow \mathbb{R}$ by

$$
u_{1}(x):=\frac{1}{\lambda^{\mu / 2}}\left(u(\lambda x)-a_{0}\right) .
$$

From Lemma 7. $f_{B_{1}} u_{1}^{2} \omega(x) d x \leq 1$ and arguing as at the beginning of this Section, we deduce $u_{1}$ is a local minimizer of

$$
\tilde{J}(v):=\int \tilde{\omega}(x)|\nabla v|^{2}+\varepsilon \lambda^{2(1-\mu / 2)} \chi_{\left\{v>\lambda^{\mu / 2} a_{0}\right\}} d x,
$$

where $\tilde{\omega}$ is a weight in the same $A_{2}$ class as $\omega$ and satisfies (H1) with the same constant $\tau_{0}$. Because $0<\mu<1, \varepsilon \lambda^{2(1-\mu / 2)}<\varepsilon$, we can argue as in Lemma 7 to find another constant $\left|a_{1}\right|<\Lambda$, such that

$$
f_{B_{\lambda}}\left|u_{1}-a_{1}\right|^{2} \omega(x) d x \leq \lambda^{\mu}
$$

which yields for $u$ the estimate

$$
f_{B_{\lambda^{2}}}\left|u-\left(a_{0}+\lambda^{\mu / 2} a_{1}\right)\right|^{2} \omega(x) d x \leq \lambda^{2 \mu} .
$$

Arguing now by induction, we conclude

$$
f_{B_{\lambda^{n}}}\left|u-a_{n}\right|^{2} \omega(x) d x \leq \lambda^{n \cdot \mu} .
$$

where

$$
a_{n}=a_{0}+\lambda^{\mu / 2} a_{1}+\cdots+\lambda^{(n-1) \mu / 2} a_{n-1} .
$$

One easily verifies that the above is a Cauchy sequence, and therefore convergent. Let $a_{\infty}=\lim _{n \rightarrow \infty} a_{n}$. We estimate

$$
\left|a_{\infty}-a_{n}\right| \leq \lim _{m \rightarrow \infty} \Lambda \cdot \sum_{i=n}^{m} \lambda^{(\mu / 2) i} \leq \frac{\Lambda}{1-\lambda^{\mu / 2}} \cdot \lambda^{n \mu / 2}
$$


We can therefore estimate, from (4.8) and (4.9)

$$
f_{B_{\lambda^{n}}}\left|u-a_{\infty}\right|^{2} \omega(x) d x \leq\left(1+\left[\frac{\Lambda}{1-\lambda^{\mu / 2}}\right]^{2}\right) \lambda^{n \cdot \mu} .
$$

Now, fixing $y \in B_{1 / 2}$ and working with $v(x)=u(y+x)$, we reach (4.10) for $B_{\lambda^{n}}(y)$. Owing to (4.10), it is easy to see that, if $u_{B_{\lambda^{n}(y)}}$ denotes $\frac{1}{\omega\left(B_{\lambda^{n}(y)}\right)} \int_{B_{\lambda^{n}(y)}} u(x) \omega(x) d x$, then

$$
\begin{aligned}
f_{B_{\lambda^{n}(y)}}\left|u-u_{B_{\lambda^{n}(y)}}\right|^{2} \omega(x) d x & \leq 2\left(f_{B_{\lambda^{n}(y)}}\left|u-a_{\infty}\right|^{2} \omega(x) d x\right. \\
& \left.+f_{B_{\lambda^{n}(y)}}\left|u_{B_{\lambda^{n}(y)}}-a_{\infty}\right|^{2} \omega(x) d x\right)
\end{aligned}
$$

Furthermore,

$$
\begin{aligned}
f_{B_{\lambda^{n}(y)}}\left|u_{B_{\lambda^{n}(y)}}-a_{\infty}\right|^{2} \omega(x) d x & =f_{B_{\lambda^{n}(y)}}\left|f_{B_{\lambda^{n}(y)}}\left(u-a_{\infty}\right) \omega(x) d x\right|^{2} \omega(x) d x \\
& \leq f_{B_{\lambda^{n}(y)}}\left|u-a_{\infty}\right|^{2} \omega(x) d x
\end{aligned}
$$

by Jensen's inequality. Thus, we conclude that

$$
f_{B_{\lambda^{n}}}\left|u-u_{B_{\lambda^{n}(y)}}\right|^{2} \omega(x) d x \leq 4\left(1+\left[\frac{\Lambda}{1-\lambda^{\mu / 2}}\right]^{2}\right) \lambda^{n \cdot \mu} .
$$

Finally, as noted earlier, the measure $A \mapsto \int_{A} \omega(x) d x$ is doubling and clearly continuous in the sense that

$$
\lim _{y \rightarrow x} \omega\left(B_{r}(x) \triangle B_{r}(y)\right)=0,
$$

for all $r>0$. Thus, applying Campanato's theorem for doubling measures, see e.g. [10, Theorem 3.2], we conclude $u$ is Hölder continuous and the proof of Theorem 8 is complete.

\section{Homogenization}

We now turn our attention to limiting free boundary problems arising from homogenization. That is, hereafter we assume

(H2) Let 0 be a free boundary point. There exists $-d<\alpha \leq 0$ such that, as $\lambda \rightarrow 0+$,

$$
\omega_{\lambda}(x):=\lambda^{|\alpha|} \omega(\lambda x)
$$

converges locally in $L^{1}$ to a weight $\omega_{0}(x) \in A_{2}$.

As we shall demonstrate, condition (H2) yields an elegant solution to the tantalizing question raised in the introduction on geometric classification of the behavior of $u$ at distinguished free boundary points. When (H2) holds at

a free boundary point, 0 , we will say that 0 is a singular free boundary point 
of degree $|\alpha|$. In particular, regular free boundary points from classical study of cavitation problems, i.e. $\omega(0)$ finite, represent (singular) free boundary points of degree zero.

The weight $\omega_{0}$ is the homogenization limit, as it verifies $\omega_{0}(t x)=t^{\alpha} \omega_{0}(x)$, for all $t>0$. Of course, should the original weight $\omega$ be homogeneous, then (H2) is immediately verified.

The examples discussed at the end of Section 2 are, essentially, homogeneous. In addition to classical homogenization procedures, condition (H2) further contemplates perturbations of those by terms $g(x)=\mathrm{o}\left(|x|^{-|\alpha|}\right)$ as $|x| \rightarrow 0$, and products by bounded, positive functions $\theta(x) \in \mathrm{VMO}$. So typically, we have in mind weights of the form

$$
\omega(x)=\theta(x) \omega_{0}(x)+g(x),
$$

where $\omega_{0}$ is $\alpha$-homogeneous (as in the examples from Section 2), $\theta \in \mathrm{VMO}$ verifying $0<\lambda_{0}<\theta(x)<\lambda_{0}^{-1}$, and $|x|^{|\alpha|} g(x) \rightarrow 0$ as $|x| \rightarrow 0$.

Lemma 9. Assume condition (H2) is in order. There exist constants $0<$ $\tau_{\star} \leq L<\infty$ such that

$$
\tau_{\star} r^{\alpha} \leq f_{B_{r}(0)} \omega(x) d x \leq L r^{\alpha}
$$

or all $0<r \ll 1$.

Proof. For each $0<r \ll 1$ define the weight $\omega_{r}: B_{1} \rightarrow \mathbb{R}$ as $\omega_{r}(y)=$ $r^{|\alpha|} \omega(r y)$. It follows from condition (H2) that $\left\{\omega_{r}(y)\right\}_{r>0}$ is a bounded set in $L^{1}\left(B_{1}\right)$. Hence, for some $L>0$,

$$
L \geq f_{B_{1}(0)} \omega_{r}(x) d x=r^{|\alpha|} f_{B_{r}(0)} \omega(x) d x
$$

which shows the estimate from above. Now, let's suppose, seeking a contradiction, that no such a $\tau_{\star}>0$ exists. It means one could find a sequence of radii $r_{j}=\mathrm{o}(1)$ such that

$$
f_{B_{r_{j}}(0)} \omega(x) d x \leq 2^{-j} r_{j}^{|\alpha|} .
$$

However, this would imply $\omega_{r_{j}}(y)=r_{j}^{|\alpha|} \omega\left(r_{j} y\right) \rightarrow 0$ in $L^{1}$, which contradicts condition (H2), as $0 \notin A_{2}$.

Before we continue, let us make a comment on the scaling of the free boundary problem (2.2), which further substantiates (H2).

Remark 10. Let $u$ be a local minimizer of

$$
\mathcal{J}(\omega, u, \Omega):=\int_{\Omega} \omega(x)|\nabla u(x)|^{2}+\chi_{\{u>0\}} d x .
$$


Given $0<\lambda<1$, define

$$
\beta=1-\frac{\alpha}{2}, \quad u_{\lambda}(x)=\lambda^{-\beta} u(\lambda x), \quad \text { and } \quad \omega_{\lambda}(x)=\lambda^{|\alpha|} \omega(\lambda x),
$$

then change of variables yields

$$
\begin{aligned}
\mathcal{J}(\omega, u, \Omega) & =\int_{\Omega / \lambda}\left[\omega(\lambda y)|\nabla u(\lambda y)|^{2}+\chi_{\{u(\lambda y)>0\}}\right] \lambda^{d} d y \\
& =\int_{\Omega / \lambda}\left[\lambda^{\alpha} \omega_{\lambda}(y) \lambda^{2(-1+\beta)}\left|\nabla u_{\lambda}(y)\right|^{2}+\chi_{\left\{u_{\lambda}(y)>0\right\}}\right] \lambda^{d} d y \\
& =\lambda^{d} \mathcal{J}\left(\omega_{\lambda}, u_{\lambda}, \Omega / \lambda\right) .
\end{aligned}
$$

That is, $u_{\lambda}$ is a local minimizer of functional $\mathcal{J}_{\lambda}$, ruled by an approximation of the homogenizing medium.

In the sequel we indeed show that local minima of the functional $\mathcal{J}_{\lambda}$ converge to a minimizer of the singular homogenized problem ruled by $\omega_{0}$.

Theorem 11. Assume 0 is a free boundary point and that $\omega$ satisfies (H1) and (H2). Let $\lambda_{k}$ be any sequence converging to zero and $u_{k}$ local minima of $\mathcal{J}_{k}=\mathcal{J}\left(\omega_{k}, v, \Omega\right)$, with $\omega_{k}(x):=\lambda_{k}^{|\alpha|} \omega\left(\lambda_{k} x\right)$. Then, up to a subsequence, $u_{k}$ converges locally uniformly to a local minimum of $\mathcal{J}\left(\omega_{0}, v, \Omega\right)$.

Proof. From Theorem 8 , minimization properties and (H1), up to a subsequence, $u_{k} \rightarrow u_{0}$ locally uniformly in $\Omega$ and locally weakly in $W^{1,2}$. Also, from (H2), $\omega_{k}^{1 / 2} \rightarrow \omega_{0}^{1 / 2}$ strongly in $L^{2}$, and thus

$$
\omega_{k}^{1 / 2} \nabla u_{k} \rightarrow \omega_{0}^{1 / 2} \nabla u_{0},
$$

locally in the weak topology of $L^{2}$. Lower weak semicontinuity of norm along with Fatou's Lemma imply, for any fixed subdomain $\Omega^{\prime} \Subset \Omega$,

$$
\mathcal{J}\left(\omega_{0}, u_{0}, \Omega^{\prime}\right) \leq \liminf \mathcal{J}\left(\omega_{k}, u_{k}, \Omega^{\prime}\right) .
$$

Now, fix a ball $B:=B_{r}\left(x_{0}\right) \Subset \Omega$ and let $\varphi$ be a smooth function in $B$ satisfying $\varphi=u_{0}$ on $\partial B$. For $\varepsilon>0$ small, consider

$$
\psi(y):=\frac{\left|y-x_{0}\right|-r}{\varepsilon r}
$$

and define $\varphi_{k}^{\varepsilon}: \Omega \rightarrow \mathbb{R}$ to be the linear interpolation between $u_{k}$ and $\varphi$ within $B_{(1+\varepsilon) r}\left(x_{0}\right)$, that is:

$$
\varphi_{k}^{\varepsilon}(y)= \begin{cases}\varphi(y), & \text { if }\left|y-x_{0}\right| \leq r \\ u_{k}(y), & \text { if }\left|y-x_{0}\right| \geq(1+\varepsilon) r \\ (1-\psi(y)) u_{0}(y)+\psi(y) u_{k}(y), & \text { if } r<\left|y-x_{0}\right|<(1+\varepsilon) r\end{cases}
$$

Since $u_{k}$ is a local minimizer of $\mathcal{J}_{k}$ over $\widetilde{B}:=B_{(1+\varepsilon) r}\left(x_{0}\right)$, we have

$$
\mathcal{J}\left(\omega_{k}, u_{k}, \widetilde{B}\right) \leq \mathcal{\partial}\left(\omega_{k}, \varphi_{k}^{\varepsilon}, \widetilde{B}\right)
$$


that is

$$
\mathcal{J}\left(\omega_{k}, u_{k}, \widetilde{B}\right) \leq \mathcal{J}\left(\omega_{k}, \varphi, B\right)+\mathcal{J}\left(\omega_{k}, \varphi_{k}^{\varepsilon}, \widetilde{B} \backslash B\right) .
$$

On the transition region, $r<\left|y-x_{0}\right|<(1+\varepsilon) r$, taking into account (5.3), we compute, for a.e. $y$,

$$
\nabla \varphi_{k}^{\varepsilon}(y)=\nabla \psi(y) \cdot\left(u_{k}(y)-u_{0}(y)\right)+(1-\psi(y)) \nabla u_{0}(y)+\psi(y) \nabla u_{k}(y)
$$

which yields

$$
\left|\nabla \varphi_{k}^{\varepsilon}(y)\right| \leq\left|\nabla u_{0}(y)\right|+\left|\nabla u_{k}(y)\right|+\frac{C}{\varepsilon}\left|u_{k}(y)-u_{0}(y)\right| .
$$

Consequently, taking into account that $|\widetilde{B} \backslash B| \sim \varepsilon$, that $\omega_{k}(y)\left|\nabla u_{k}\right|^{2}$ is bounded in $L^{1+\delta}(\widetilde{B})$, for some $\delta>0$, and that $\omega_{k}(y)$ is bounded in $L^{1}$, we obtain, from Hölder inequality

$$
\begin{aligned}
\mathcal{J}\left(\omega_{k}, \varphi_{k}^{\varepsilon}, \widetilde{B} \backslash B\right) & =\int_{\tilde{B} \backslash B} \omega_{k}(x)\left|\nabla \varphi_{k}^{\varepsilon}\right|^{2}+\chi_{\left\{\varphi_{k}^{\varepsilon}>0\right\}} d x \\
& \leq C_{1}|\widetilde{B} \backslash B|^{\frac{\delta}{1+\delta}}+C_{2}\left(\frac{C}{\varepsilon}\right)^{2}\left\|u_{k}-u_{0}\right\|_{L^{\infty}(\widetilde{B} \backslash B)}+\varepsilon \\
& \leq C \varepsilon^{\beta}+\varepsilon^{-2} \mathrm{O}(1),
\end{aligned}
$$

where $0<\beta=\delta /(1+\delta) \ll 1$, is a small but positive number and $\mathrm{o}(1)=$ $C\left\|u_{k}-u_{0}\right\|_{L^{\infty}(\widetilde{B} \backslash B)}$ is an error that goes to zero as $k \rightarrow \infty$. Hence, combining (5.3), (5.4) and (5.6),

$$
\mathcal{J}\left(\omega_{k}, u_{k}, \widetilde{B}\right) \leq \mathcal{J}\left(\omega_{k}, \varphi, B\right)+C \varepsilon^{\beta}+\varepsilon^{-2} \mathrm{o}(1),
$$

as $k \rightarrow \infty$. Finally, letting $k \rightarrow \infty$, taking into account (5.2), we obtain

$$
\mathcal{J}\left(\omega_{0}, u_{0}, \tilde{B}\right) \leq \mathcal{J}\left(\omega_{0}, \varphi, B\right)+C \varepsilon^{\beta} .
$$

Since $\varphi$ and $\varepsilon>0$ were taken arbitrary, we conclude the proof of the Theorem.

\section{6. $C^{1+\gamma}$ REGULARITY AT THE FREE BOUNDARY}

In Section 4 we showed minimizers are locally Hölder continuous, which, in particular, yields a rough oscillation control of $u$ near the free boundary. The heart of the matter, though, is to describe the precise geometric behavior of a local minimizer at free boundary points. Roughly speaking, solutions to singular free boundary problems should adjust their vanishing rate based upon the singularity of the medium.

While there is no hope to obtain an estimate superior than Hölder continuity of local minima, at any other point, in this section we show that $u$ behaves as a $C^{1+\gamma}$ function around a singular free boundary point. As usual, this implies higher order differentiability of $u$ at free boundary points. In particular, if $1+\gamma=N$ is an integer, then $u \in C^{N-1,1}$, in the sense it is $N-1$ differentiable, and the $N$ th-Newtonian quotient remains bounded. 
When $1+\gamma=N+\theta$, for $0<\theta<1$, then solutions are $C^{N, \theta}$ regular at free boundary points - see [18] for similar higher differentiability phenomenon.

This is the content of the following key result:

Theorem 12. Assume $(H 1)-(H 2)$ and let $u$ be a local minimizer of (2.2) so that 0 is a free boundary point. Then

$$
\sup _{B_{r}} u \leq C r^{1+\frac{|\alpha|}{2}},
$$

for a universal constant $C>0$, independent of $u$.

The proof of Theorem 12 will be based on a geometric flatness improvement technique, in the spirit of [15, 17]. For that, we need:

Lemma 13. Assume $(H 1)-(H 2)$ and let $u \in H^{1}\left(B_{1}, \omega\right)$ verify $0 \leq u \leq 1$ in $B_{1}$, with $u(0)=0$. Given $\delta>0$, there exists $\varepsilon>0$ depending only on $\delta$ and universal constants, such that if $u$ is a local minimizer of

$$
\partial_{\varepsilon}(u):=\int_{B_{1}} \omega_{\lambda}(x)|\nabla u(x)|^{2}+\varepsilon \chi_{\{u>0\}} d x,
$$

for any $0<\lambda<1$, then, in $B_{1 / 2}, u$ is at most $\delta$, that is,

$$
\sup _{B_{1 / 2}} u \leq \delta .
$$

Proof. Suppose, for the sake of contradiction, that the thesis of the Lemma does not hold. This means for some $\delta_{0}>0$, one can find a sequence of functions $u_{j} \in H^{1}\left(B_{1}, \omega_{j}\right)$ satisfying:

(1) $0 \leq u_{j} \leq 1$ in $B_{1}$;

(2) $u_{j}(0)=0$

(3) $u_{j}$ is a local minimizer of $\mathcal{J}_{j}(u):=\int_{B_{1}} \omega_{\lambda_{j}}(x)|\nabla u(x)|^{2}+\frac{1}{j} \chi_{\{u>0\}} d x$; however,

$$
\sup _{B_{1 / 2}} u_{j} \geq \delta_{0} \quad \forall j \in \mathbb{N} .
$$

From compactness estimates proven in Section 4, up to a subsequence, $u_{j}$ converges locally uniformly to a nonnegative function $u_{\infty}$, with $u_{\infty}(0)=0$. Also, from similar analysis as in Theorem 11, $u_{\infty}$ is a local minimizer of

$$
\mathcal{J}_{0}(v):=\int_{B_{1}} \omega_{0}(x)|\nabla v(x)|^{2} d x .
$$

Applying maximum principle (available for minimizers of the functional $\partial_{0}$ ), we conclude $u_{\infty} \equiv 0$. That is, we have proven $u_{j}$ converges locally uniformly to 0 . Therefore, for $j \gg 1$, we reach a contradiction with (6.3). The proof of Lemma 13 is complete.

Proof of Theorem 12. We will make few (universal) decisions. Initially we set

$$
\delta_{\star}:=2^{\frac{\alpha}{2}-1} .
$$


Lemma 13 assures the existence of a positive (universal) constant $\varepsilon_{0}>0$ such that any normalized minimizer of $\partial_{\varepsilon_{0}}$, as defined in (6.1) verifies (6.2), for $\delta_{\star}$. Define

$$
\varrho_{0}:=\sqrt[2-\alpha]{\varepsilon_{0}}, \quad \text { and } \quad \tilde{u}(x):=u\left(\varrho_{0} x\right) .
$$

By remark 10, $\tilde{u}$ is a local minimizer of $\partial_{\varepsilon_{0}}$, and hence, from Lemma 13, there holds:

$$
\sup _{B_{1 / 2}} \tilde{u}(x) \leq 2^{\frac{\alpha}{2}-1} .
$$

Next, by induction, we iterate the previous argument to show

$$
\sup _{B_{2}-k} \tilde{u}(x) \leq 2^{k\left(\frac{\alpha}{2}-1\right)} .
$$

Estimate (6.4) gives the first step of induction, $k=1$. Now, suppose we have verified (6.5) for $k=1,2, \cdots p$. Define

$$
\tilde{v}(x):=2^{p\left(1-\frac{\alpha}{2}\right)} \cdot \tilde{u}\left(2^{-p} x\right) .
$$

It follows from induction hypothesis that $0 \leq v \leq 1$. Also, from scaling, we check that $\tilde{v}$ is also a minimizer of $\partial_{\varepsilon_{0}}$. Applying Lemma 13 to $\tilde{v}$ we conclude

$$
\sup _{B_{1 / 2}} \tilde{v} \leq 2^{\frac{\alpha}{2}-1}
$$

which, in terms of $\tilde{u}$, gives precisely the $(p+1)$ step of induction.

Now, given a (universally small) radius $r>0$, choose $k \in \mathbb{N}$, such that

$$
2^{-(k+1)}<\varrho_{0}^{-1} r \leq 2^{-k}
$$

We can then estimate

$$
\begin{aligned}
\sup _{B_{r}} u & =\sup _{B_{\varrho_{0}^{-1} r}} \tilde{u} \\
& \leq \sup _{B_{2}-k} \tilde{u} \\
& \leq 2^{k\left(\frac{\alpha}{2}-1\right)} \\
& \leq\left(\frac{2}{\varrho_{0}}\right)^{1-\frac{\alpha}{2}} \cdot r^{1+\frac{|\alpha|}{2}} \\
& =C r^{1+\frac{|\alpha|}{2}},
\end{aligned}
$$

for $C>1$ universal, as required.

\section{NONDEGENERACY AND WEAK GEOMETRY}

In the previous section we show local minima are $C^{1+\gamma}$ smooth along the singular free boundary, for $\gamma=\frac{|\alpha|}{2}$. In this section we prove a competing inequality which assures that such a geometric decay is sharp. Surprisingly enough, for such an estimate one only needs the corresponding upper bound for the degree of singularity of the free boundary and not the full condition 
(H2). Thus, for didactical purposes we state it as a separate condition, which, in accordance to Lemma 9, is implied by condition (H2).

(H3) For some $-d<\alpha \leq 0$, there exists $L>0$ such that

$$
f_{B_{r}(0)} \omega(x) d x \leq L r^{\alpha}
$$

for all $0<r \ll 1$.

Again, we recall 0 is the localized free boundary point at where we are analyzing the geometric behavior of $u$. Thus, condition (H3) simply conveys the idea that the origin is a singular free boundary point of degree at most $|\alpha|$. Note that (H3) yields

$$
\sup _{r>0} f_{B_{1}} \omega_{r}(x) d x \leq L,
$$

where, as before, $\omega_{r}(x)=r^{|\alpha|} \omega(r x)$.

Theorem 14. Let $u$ be a minimizer of (2.2), 0 a free boundary point and assume (H3). Then

$$
\sup _{B_{r}} u(x) \geq 2 \cdot \sqrt{\frac{1}{L} \frac{d^{d}}{(d+2)^{d+2}}} \cdot r^{1+\frac{|\alpha|}{2}}, \quad \forall 0<r<1,
$$

where $d$ is dimension.

Proof. The idea of the proof is to cut a family of concentric holes on the graph of $u$, compare the resulting functions with $u$ in terms of the minimization problem $\mathcal{J}$, and finally optimize the cutting-hole parameter; here are the details. Let $0<r<1$ be a fixed radius and define $v_{r}: B_{1} \rightarrow \mathbb{R}$ as

$$
v_{r}(y):=r^{\frac{\alpha}{2}-1} u(r y) \text {. }
$$

The goal is to show that

$$
\mathcal{S}_{r}:=\sup _{B_{1}} v_{r}
$$

is uniformly bounded from below, independently of $r$. From Remark 10, $v_{r}$ is a local minimizer of $\mathcal{J}_{r}$ over $B_{1}$. Next, let us choose $0<\sigma<1,0<\varepsilon \ll 1$ and craft a smooth, radially symmetric function $\varphi: B_{1} \rightarrow \mathbb{R}$ satisfying:

$$
0 \leq \varphi \leq 1, \quad \varphi \equiv 0 \text { in } B_{\sigma}, \quad \varphi=1 \text { on } \partial B_{1}, \quad|\nabla \varphi| \leq(1+\varepsilon)(1-\sigma)^{-1} .
$$

In the sequel, let us consider the test function $\xi: B_{1} \rightarrow \mathbb{R}$ given by

$$
\xi(x):=\min \left\{v_{r}(x),(1+\varepsilon) \mathcal{S}_{r} \cdot \varphi(x)\right\} .
$$

By construction, $\xi$ competes with $v_{r}$ in the minimization problem $\partial_{r}$, and thus

$$
\int_{B_{1}}\left(\omega_{r}(x)|\nabla \xi|^{2}+\chi_{\{\xi>0\}}\right) d x \geq \int_{B_{1}}\left(\omega_{r}(x)\left|\nabla v_{r}\right|^{2}+\chi_{\{v>0\}}\right) d x .
$$


We can rewrite (7.3) as an inequality of the form $\mathcal{A} \geq \mathcal{B}$, for

$$
\begin{aligned}
& \mathcal{A}:=\int_{B_{1}} \omega_{r}(x)\left(|\nabla \xi|^{2}-|\nabla v|^{2}\right) d x, \\
& \mathcal{B}:=\int_{B_{1}}\left(\chi_{\left\{v_{r}>0\right\}}-\chi_{\{\xi>0\}}\right) d x .
\end{aligned}
$$

As to estimate $\mathcal{B}$ from below we note that $\{v>0\} \supset\{\xi>0\}$, thus

$$
\mathcal{B}=\int_{B_{1}} \chi_{\{\xi=0\}} d x \geq\left|B_{\sigma}\right| .
$$

Next we estimate $\mathcal{A}$ from above. For that, let us define

$$
\Pi:=\left\{x \in B_{1} \mid(1+\varepsilon) \mathcal{S}_{r} \cdot \varphi(x)<v_{r}(x)\right\}
$$

and compute

$$
\begin{aligned}
\int_{B_{1}} \omega_{r}(y)\left(|\nabla \xi|^{2}-\left|\nabla v_{r}\right|^{2}\right) d x & =\int_{\Pi} \omega_{r}(x)\left(|\nabla \xi|^{2}-\left|\nabla v_{r}\right|^{2}\right) d x \\
& \leq(1+\varepsilon)^{2} \mathcal{S}_{r}^{2} \int_{B_{1}} \omega_{r}(x)|\nabla \varphi|^{2} d x \\
& \leq(1+\varepsilon)^{4}(1-\sigma)^{-2} \cdot\left(\int_{B_{1}} \omega_{r}(x) d x\right) \cdot \mathcal{S}_{r}^{2} \\
& \leq(1+\varepsilon)^{4}(1-\sigma)^{-2} \cdot L\left|B_{1}\right| \cdot \mathcal{S}_{r}^{2},
\end{aligned}
$$

where in the last inequality we have used condition (H3), along with comment (7.1). From the relation $\mathcal{A} \geq \mathcal{B}$, we obtain

$$
\begin{aligned}
\mathcal{S}_{r}^{2} & \geq \frac{\left|B_{\sigma}\right|}{L\left|B_{1}\right|} \cdot \frac{\left(1-\sigma^{2}\right)}{(1+\varepsilon)^{4}} \\
& =\frac{1}{L(1+\varepsilon)^{4}} \cdot \frac{\sigma^{d}}{\left(1-\sigma^{2}\right)}
\end{aligned}
$$

Letting $\varepsilon \rightarrow 0$ and selecting $\sigma=\frac{d}{d+2}$ yields the optimal lower bound. The proof of Theorem 14 is complete.

An important consequence of Theorem 12 and Theorem 14 combined is that, around free boundary points of same homogeneity $\alpha$, a local minimum detaches from its coincidence set, $\{u=0\}$, precisely as dist ${ }^{1+\frac{|\alpha|}{2}}$.

Corollary 15. Let $u$ be a minimizer of (2.2) and assume all free boundary points in $B_{1 / 2}$ satisfy conditions (H1) and (H2). Then there exists a universal constant $C>1$ such that

$$
C^{-1} \operatorname{dist}(x, \partial\{u>0\})^{1+\frac{|\alpha|}{2}} \leq u(x) \leq C \cdot \operatorname{dist}(x, \partial\{u>0\})^{1+\frac{|\alpha|}{2}}
$$

for any $x \in B_{1 / 4} \cap\{u>0\}$. 
By standard arguments we then conclude that near free boundary points of same homogeneity, the set of positivity $\{u>0\}$ has uniform positive density:

Corollary 16. Let $u$ be a minimizer of (2.2) and assume all free boundary points in $B_{1 / 2}$ satisfy (H1) and (H2). Then, for any $0<r<\frac{1}{4}$,

$$
\frac{\left|B_{r} \cap\{u>0\}\right|}{\left|B_{r}\right|} \geq C,
$$

where $0<C<1$ is a universal constant, independent of $r$.

\section{REFERENCES}

[1] Caffarelli, L. and Alt, H. Existence and regularity for a minimum problem with free boundary. (1981) J. Reine Angew. Math. 325, 105-144.

[2] Caffarelli, L., Roquejoffre, J-M and Sire, Y. Variational problems for free boundaries for the fractional Laplacian. J. Eur. Math. Soc. (JEMS) 12 (2010), no. 5, 1151-1179.

[3] Caffarelli, L. and Silvestre, L. An extension problem related to the fractional Laplacian. Comm. Partial Differential Equations 32 (2007), no. 7-9, 1245-1260.

[4] De Silva, D. Free boundary regularity for a problem with right hand side. Interfaces Free Bound. 13 (2011), no. 2, 223-238.

[5] Dos Prazeres, D. and Teixeira, E. Cavity problems in discontinuous media. Calc. Var. Partial Differential Equations 55 (2016), no. 1, Art. 10, 15 pp.

[6] Fabes, E., Kenig, C. and Serapioni, R. The local regularity of solutions of degenerate elliptic equations. Comm. Partial Differential Equations 7 (1982), no. 1, 77-116.

[7] Fabes, E., Jerison, D. and Kenig, C. The Wiener test for degenerate elliptic equations. Ann. Inst. Fourier (Grenoble) 32 (1982), no. 3, vi, 151-182.

[8] Fabes, E., Jerison, D. and Kenig, C. Boundary behavior of solutions to degenerate elliptic equations. Conference on harmonic analysis in honor of Antoni Zygmund, Vol. I, II (Chicago, Ill., 1981), 577-589,

[9] Ferrari, F.; Salsa, S. Regularity of the free boundary in two-phase problems for linear elliptic operators. Adv. Math. 214 (2007), no. 1, 288-322.

[10] Górka, Przemyslaw Campanato theorem on metric measure spaces. Ann. Acad. Sci. Fenn. Math. 34 (2009), no. 2, 523-528.

[11] Heinonen, Juha; Kilpeläinen, Tero; Martio, Olli Nonlinear potential theory of degenerate elliptic equations. Unabridged republication of the 1993 original. Dover Publications, Inc., Mineola, NY, 2006. xii+404 pp. ISBN: 0-486-45050-3

[12] Moreira, D.; Teixeira, E. On the behavior of weak convergence under nonlinearities and applications. Proc. Amer. Math. Soc. 133 (2005), no. 6, 1647-1656.

[13] Muckenhoupt, B Weighted norm inequalities for the Hardy maximal function. Trans. Amer. Math. Soc. (1972) 165 207-226.

[14] Teixeira, E. A variational treatment for general elliptic equations of the flame propagation type: regularity of the free boundary. Ann. Inst. H. Poincar Anal. Non Linéaire 25 (2008), no. 4, 633-658.

[15] Teixeira, E. Regularity for quasilinear equations on degenerate singular sets. Math. Ann. 358 (2014), no. 1-2, 241-256.

[16] Teixeira, E. Sharp regularity for general Poisson equations with borderline sources. J. Math. Pures Appl. (9) 99 (2013), no. 2, 150-164.

[17] Teixeira, E. Universal moduli of continuity for solutions to fully nonlinear elliptic equations. Arch. Ration. Mech. Anal. 211 (2014), no. 3, 911-927. 
[18] Teixeira, E. Regularity for the fully nonlinear dead-core problem. Math. Ann. 364 (2016), no. 3-4, 1121-1134.

Sorbonne Université, Université Paris Diderot, CNRS, Institut de Mathématiques De Jussieu-Paris Rive Gauche, IMJ-PRG, F-75005, Paris, France

E-mail address: jimmy.lamboley@imj-prg.fr

Johns Hopkins University, Krieger Hall, N. Charles St., Baltimore, MD 21218

E-mail address: sire@math.jhu.edu

University of Central Florida, 4393 Andromeda loop N, Orlando, FL 32816

E-mail address: eduardo.teixeira@ucf.edu 